ESAIM: COCV 27 (2021) 37

https://doi.org/10.1051/cocv/2021040
ESAIM: Control, Optimisation and Calculus of Variations

www.esaim-cocv.org

\title{
SPACE OF SIGNATURES AS INVERSE LIMITS OF CARNOT GROUPS*
}

\author{
ENRICO Le DONnE ${ }^{1,2, * *}$ AND Roger Züst ${ }^{3}$
}

\begin{abstract}
We formalize the notion of limit of an inverse system of metric spaces with 1-Lipschitz projections having unbounded fibers. The construction is applied to the sequence of free Carnot groups of fixed rank $n$ and increasing step. In this case, the limit space is in correspondence with the space of signatures of rectifiable paths in $\mathbb{R}^{n}$, as introduced by Chen. Hambly-Lyons's result on the uniqueness of signature implies that this space is a geodesic metric tree. As a particular consequence we deduce that every path in $\mathbb{R}^{n}$ can be approximated by projections of some geodesics in some Carnot group of rank $n$, giving an evidence that the complexity of sub-Riemannian geodesics increases with the step.
\end{abstract}

Mathematics Subject Classification. 22E25, 53C17, 49Q15, 28A75, 60H05.

Received April 29, 2020. Accepted April 5, 2021.

\section{INTRODUCTION}

In the study of rough paths in stochastic PDE theory to each path one assigns its signature via the method of iterated integrals. This concept was introduced by Chen $[4,5]$ who aimed to give an algebraic structure to the space of paths. The space of $k$-truncated signatures of rectifiable paths in $\mathbb{R}^{n}$ has the structure of the free nilpotent Lie group of rank $n$ and step $k$. In fact, the truncated signatures have a sub-Riemannian meaning. Namely, the above Lie group has a natural structure of a Carnot group and hence to every rectifiable path in $\mathbb{R}^{n}$ one assigns a horizontal lift starting from the identity in the Carnot group. The truncated signature of the path is the final point of the lifted path.

With the idea that the signature of a rectifiable path lies in an infinite dimensional tensor algebra, one would like to metrize the space of signatures as a limit of Carnot groups. The first aim of this paper is to formalize the appropriate notion of limit of inverse systems of metric spaces. Our construction differs from previous ones as for example in [6] by the fact that the fibers of the bonding maps may not have finite diameter and it is inspired by the construction of ultralimits of metric spaces. Namely, let $\left(\left(X_{i}, \star_{i}\right)_{i \in I},\left(\pi_{j}^{i}\right)_{i \geq j \in I}\right)$ be an inverse system of pointed metric spaces and 1-Lipschitz base-point-preserving maps $\pi_{j}^{i}:\left(X_{i}, \star_{i}\right) \rightarrow\left(X_{j}, \star_{j}\right)$ as

*E.L.D. was partially supported by the Academy of Finland (grant 288501 'Geometry of subRiemannian groups' and by grant 322898 'Sub-Riemannian Geometry via Metric-geometry and Lie-group Theory') and by the European Research Council (ERC Starting Grant 713998 GeoMeG 'Geometry of Metric Groups').

Keywords and phrases: Signature of paths, inverse limit, path lifting property, submetry, metric tree, Carnot group, free nilpotent group, sub-Riemannian distance.

${ }^{1}$ Dipartimento di Matematica, Università di Pisa, Largo B. Pontecorvo 5, 56127 Pisa, Italy.

2 University of Jyväskylä, Department of Mathematics and Statistics, P.O. Box (MaD), 40014, Finland.

3 Mathematical Institute, University of Bern, Alpeneggstrasse 22, 3012 Bern, Switzerland.

** Corresponding author: ledonne@msri.org 
connecting morphisms. In Lemma 2.3 we show that the system admits an inverse limit $\left(\left(X_{\infty}, \star_{\infty}\right),\left(\pi_{i}^{\infty}\right)_{i \in I}\right)$ in this category and points in $X_{\infty}$ can be characterized by those elements $\left(x_{i}\right)_{i \in I} \in \prod_{i \in I} X_{i}$ such that

$$
\pi_{j}^{i}\left(x_{i}\right)=x_{j} \text { for all } i \geq j \text { and } \sup _{i \in I} d\left(\star_{i}, x_{i}\right)<\infty
$$

Our interest will be in the inverse limit of the system $\left(G^{k}\left(\mathbb{R}^{n}\right), \pi_{k}\right)_{k \in \mathbb{N}}$, where $G^{k}\left(\mathbb{R}^{n}\right)$ is the free Carnot group of rank $n$ and step $k$, pointed at the identity element. The map $\pi_{k}: G^{k}\left(\mathbb{R}^{n}\right) \rightarrow G^{k-1}\left(\mathbb{R}^{n}\right)$ is the projection modulo the $k$-layer, i.e., the $k$-th element of the lower central series of $G^{k}\left(\mathbb{R}^{n}\right)$. The maps $\pi_{k}$ have two properties that will be crucial in our following discussion: they are submetries (see Def. 2.1) and have lifting properties for rectifiable paths (see Defs. 2.5 and 2.8). Under this type of assumptions we can draw several conclusions on our inverse limit space $X_{\infty}$. For example, also the projections maps $\pi_{i}^{\infty}$ are submetries with lifting properties and the metric space $X_{\infty}$ is a geodesic space, if so are the $X_{i}$ 's (see Lem. 2.7 and Prop. 2.9, respectively).

We deduce that the space of signatures of rectifiable paths in $\mathbb{R}^{n}$ has a canonical identification with the metric space

$$
\lim _{k \in \mathbb{N}}\left(G^{k}\left(\mathbb{R}^{n}\right), \pi_{k}\right)
$$

We draw some consequences from a deep result of Hambly and Lyons [8] on the uniqueness of signatures, i.e., every rectifiable path in $\mathbb{R}^{n}$ is completely determined by its signature, up to reparametrization, translation, and tree-like reduction. In Theorem 4.4 we show that the space (1.1) is a geodesic metric tree. In fact, it is a metric space homeomorphic to a tree that branches at every point with infinite valence. Further we deduce a particular statement about the complexity of sub-Riemannian geodesics in Carnot groups. Indeed, we come to the conclusion that every path in $\mathbb{R}^{n}$ can be arbitrarily approximated by the projection of some geodesic in $G^{k}\left(\mathbb{R}^{n}\right)$ for some $k$ (see Cor. 4.5).

One more purpose to study the space (1.1) was with the aim to properly define and metrize the free Lie group with $n$ generators. Clearly, the space (1.1) still has an induced group structure for which the distance is left invariant (we initially take Carnot groups with left invariant distances). Unfortunately, this space is not a topological group since the right translations are not continuous anymore. We prove this last claim in general. Namely, a group with the topology of a tree cannot have continuous left and right translations simultaneously, unless it is $\mathbb{R}$ (see Thm. 5.1).

\section{SubMETRIES AND PATH LIFTING PROPERTIES}

Let $(I, \geq)$ be a partially ordered set. For each $i \in I$, let $X_{i}$ be a metric space and let $\star_{i} \in X_{i}$ be a choice of a base point in $X_{i}$. We shall say that $\left(X_{i}, \star_{i}\right)$ is a pointed metric space. For all $i, j \in I$ with $i \geq j$ let $\pi_{j}^{i}:\left(X_{i}, \star_{i}\right) \rightarrow\left(X_{j}, \star_{j}\right)$ be a map that is 1-Lipschitz and preserves the base point, i.e., $\pi_{j}^{i}\left(\star_{i}\right)=\star_{j}$. The maps $\pi_{j}^{i}$ are called bonding maps if $\pi_{i}^{i}$ is the identity map in $X_{i}$ and $\pi_{k}^{j} \circ \pi_{j}^{i}=\pi_{k}^{i}$, for all $i \geq j \geq k$. In this case, we have that $\left(\left(X_{i}, \star_{i}\right)_{i \in I},\left(\pi_{j}^{i}\right)_{i \geq j \in I}\right)$ is an inverse system of pointed metric spaces and 1-Lipschitz base-point-preserving maps $\pi_{j}^{i}:\left(X_{i}, \star_{i}\right) \rightarrow\left(X_{j}, \star_{j}\right)$ as bonding morphisms.

The most important example for us is when $I=\mathbb{N}$. Namely, assume we have a sequence of pointed metric spaces $\left(X_{i}, d_{i}, \star_{i}\right)_{i \in \mathbb{N}}$ together with 1-Lipschitz maps $\pi_{i}: X_{i} \rightarrow X_{i-1}$ that preserve the base point. This leads to an inverse system. For $i \geq j$, we define

$$
\pi_{j}^{i}: X_{i} \rightarrow X_{j} \quad \text { as } \quad \pi_{j}^{i}:=\pi_{j+1} \circ \cdots \circ \pi_{i}
$$

As a composition of 1-Lipschitz maps, each $\pi_{j}^{i}$ is itself 1-Lipschitz. 
As discussed in the introduction, we consider the space $X_{\infty}$ as the set of elements $\left(x_{i}\right)_{i \in I} \in \prod_{i \in I} X_{i}$ with the property that $\pi_{j}^{i}\left(x_{i}\right)=x_{j}$ and

$$
\sup _{i \in I} d_{i}\left(\star_{i}, x_{i}\right)<\infty
$$

It is thus clear that $X_{\infty}$ is nonempty since $\star_{\infty}:=\left(\star_{i}\right)_{i \in I} \in X_{\infty}$. The distance between $x=\left(x_{i}\right)_{i \in I}$ and $y=\left(y_{i}\right)_{i \in I}$ in $X_{\infty}$ is defined by

$$
d_{\infty}(x, y):=\sup _{i \in I} d_{i}\left(x_{i}, y_{i}\right)
$$

There are natural projections $\pi_{i}^{\infty}: X_{\infty} \rightarrow X_{i}$ given by $\pi_{i}^{\infty}\left(\left(x_{j}\right)_{j \in I}\right):=x_{i}$ for $i \in I$.

Definition 2.1. A submetry is a 1-Lipschitz map between (nonempty) metric spaces $\pi: X \rightarrow Y$ such for any $y, y^{\prime} \in Y$ and $x \in X$ with $\pi(x)=y$ there is a $x^{\prime} \in X$ with $\pi\left(x^{\prime}\right)=y^{\prime}$ and $d_{X}\left(x, x^{\prime}\right)=d_{Y}\left(y, y^{\prime}\right)$.

If in the notation (2.1) each of the $\pi_{i}$ is a submetry, then also every $\pi_{i}^{j}$ is a submetry. This last claim follows from the simple observation that the composition of submetries is also a submetry.

Lemma 2.2. The space $\left(X_{\infty}, d_{\infty}\right)$ constructed above is a metric space and further:

(1) The projection $\pi_{i}^{\infty}:\left(X_{\infty}, d_{\infty}\right) \rightarrow\left(X_{i}, d_{i}\right)$ is 1-Lipschitz for every $i \in I$.

(2) If $X_{i}$ is complete for every $i \in I$, then so is $X_{\infty}$.

(3) If $I=\mathbb{N}$, then $d_{\infty}(x, y)=\lim _{i \rightarrow \infty} d_{i}\left(\pi_{i}^{\infty}(x), \pi_{i}^{\infty}(y)\right)$.

(4) If $I=\mathbb{N}$ and $\pi_{i}$ is a submetry for every $i$, then $\pi_{i}^{\infty}$ is a submetry for every $i \in \mathbb{N}$.

Proof. Let $x=\left(x_{i}\right)_{i \in I}, y=\left(y_{i}\right)_{i \in I}$ and $z=\left(z_{i}\right)_{i \in I}$ be arbitrary elements of $X_{\infty}$. First note that by the triangle inequality in each $X_{i}$, we have

$$
d_{\infty}(x, y) \leq \sup _{i \in I} d_{i}\left(\star_{i}, x_{i}\right)+d_{i}\left(\star_{i}, y_{i}\right) \leq \sup _{i \in I} d_{i}\left(\star_{i}, x_{i}\right)+\sup _{i \in I} d_{i}\left(\star_{i}, y_{i}\right)<\infty
$$

Hence $d_{\infty}$ is finite. Similarly one can show the triangle inequality. Indeed,

$$
\begin{aligned}
d_{\infty}(x, z) & =\sup _{i \in I} d_{i}\left(x_{i}, z_{i}\right) \leq \sup _{i \in I} d_{i}\left(x_{i}, y_{i}\right)+\sup _{i \in I} d_{i}\left(y_{i}, z_{i}\right) \\
& \leq d_{\infty}(x, y)+d_{\infty}(y, z)
\end{aligned}
$$

If $d_{\infty}(x, y)=0$, then of course $x_{i}=y_{i}$ for all $i \in I$ and hence $x=y$. Thus $d_{\infty}$ is a metric on $X_{\infty}$.

(1) is clear since $d_{i}\left(x_{i}, y_{i}\right) \leq d_{\infty}(x, y)$ for all $i \in I$.

For (2) consider a Cauchy sequence $\left(x^{n}\right)_{n \in \mathbb{N}}$ in $X_{\infty}$. We can write $x^{n}=\left(x_{i}^{n}\right)_{i \in I}$ for every $n \in \mathbb{N}$. Because $\pi_{i}^{\infty}: X_{\infty} \rightarrow X_{i}$ is 1-Lipschitz by (1), the sequence $\left(x_{i}^{n}\right)_{n \in \mathbb{N}}$ is a Cauchy sequence in $X_{i}$ for every $i \in I$. By assumption these sequences converge, say to $x_{i}:=\lim _{n \rightarrow \infty} x_{i}^{n} \in X_{i}$. We want to show that $\left(x^{n}\right)_{n \in \mathbb{N}}$ converges to $x:=\left(x_{i}\right)_{i \in I}$ in $X_{\infty}$. First note that $\pi_{j}^{i}\left(x_{i}\right)=x_{j}$ for $i \geq j$ because $\pi_{j}^{i}$ is continuous and $\pi_{j}^{i}\left(x_{i}^{n}\right)=x_{j}^{n}$ holds for all $n \in \mathbb{N}$. Because $\left(x^{n}\right)_{n \in \mathbb{N}}$ is a Cauchy sequence in $X_{\infty}$ there is some $R>0$ such that $d\left(x^{n}, \star_{\infty}\right) \leq R$ for all $n \in \mathbb{N}$, which is equivalent to $d_{i}\left(\star_{i}, x_{i}^{n}\right) \leq R$ for all $i$ and $n$. Taking limits, this implies that also $d_{i}\left(\star_{i}, x_{i}\right) \leq R$ for all $i$ and hence $x$ is an element of $X_{\infty}$. Now if $\epsilon>0$ is given, there exists $N_{\epsilon} \in \mathbb{N}$ such that $d_{\infty}\left(x^{n}, x^{m}\right) \leq \epsilon$ for all $n, m \geq N_{\epsilon}$. Again because each $\pi_{i}^{\infty}$ is 1-Lipschitz it holds that $d_{i}\left(x_{i}^{n}, x_{i}\right) \leq \epsilon$ for all $i \in I$ and all $n \geq N_{\epsilon}$. Hence

$$
d_{\infty}\left(x^{n}, x\right)=\sup _{i \in I} d_{i}\left(x_{i}^{n}, x_{i}\right) \leq \epsilon
$$


for all $n \geq N_{\epsilon}$. Thus $x^{n}$ converges to $x$ in $X_{\infty}$ and (2) follows.

To see (3) note that $\pi_{i}\left(x_{i}\right)=x_{i-1}$ and $\pi_{i}\left(y_{i}\right)=y_{i-1}$. Because each $\pi_{i}$ is 1-Lipschitz, the sequence $\left(d_{i}\left(x_{i}, y_{i}\right)\right)_{i \in \mathbb{N}}$ is increasing. Hence (3) holds.

In order to show (4) fix $k \in \mathbb{N}$ and consider $x_{k}, y_{k} \in X_{k}$ and $x=\left(x_{i}\right)_{i \in \mathbb{N}} \in X_{\infty}$, i.e., such that $\pi_{k}^{\infty}(x)=x_{k}$. For $i<k$ set $y_{i}:=\pi_{i}^{k}\left(y_{k}\right)$. For $i>k$ we recursively define $y_{i+1} \in \pi_{i+1}^{-1}\left(y_{i}\right)$ to be some point with $d_{i+1}\left(x_{i+1}, y_{i+1}\right)=$ $d_{i}\left(x_{i}, y_{i}\right)$. This is possible because each $\pi_{i}$ is a submetry. By construction and (3),

$$
\sup _{i \in \mathbb{N}} d_{i}\left(x_{i}, y_{i}\right)=\lim _{i \rightarrow \infty} d_{i}\left(x_{i}, y_{i}\right)=d_{k}\left(x_{k}, y_{k}\right)<\infty
$$

Set $y:=\left(y_{i}\right)_{i \in \mathbb{N}} \in \prod_{i \in \mathbb{N}} X_{i}$. Since

$$
\sup _{i \in \mathbb{N}} d_{i}\left(\star_{i}, y_{i}\right) \leq \sup _{i \in \mathbb{N}} d_{i}\left(\star_{i}, x_{i}\right)+\sup _{i \in \mathbb{N}} d_{i}\left(x_{i}, y_{i}\right)<\infty
$$

it follows that $y \in X_{\infty}$. From (2.2) we obtain

$$
d_{\infty}(x, y)=\lim _{i \rightarrow \infty} d_{i}\left(x_{i}, y_{i}\right)=d_{k}\left(x_{k}, y_{k}\right) .
$$

Since each $\pi_{k}^{\infty}$ is 1 -Lipschitz by (1), this shows that these maps are submetries.

We readily show that the space $\left(X_{\infty}, \star_{\infty}\right)$, as defined, is the inverse limit in the category of pointed metric spaces with base-point-preserving 1-Lipschitz maps as morphisms. Notice that we do not show the analogue considering submetries, see Example 2.4 below.

Lemma 2.3. Assume that $\left(X_{i}, \star_{i}\right)_{i \in I}$ together with $\pi_{j}^{i}: X_{i} \rightarrow X_{j}$ for $i \geq j$ is an inverse system in the category of pointed metric spaces with base-point-preserving 1-Lipschitz maps as morphisms. Then $\left(X_{\infty}, \star_{\infty}\right)$ is its inverse limit.

Proof. We need to check the universal property for $\left(X_{\infty}, \star_{\infty}\right)$. In this direction let $(Y, \star)$ be some pointed metric space together with base-point-preserving 1-Lipschitz maps $u_{i}: Y \rightarrow X_{i}$ that satisfy $\pi_{j}^{i} \circ u_{i}=u_{j}$ for all $i \geq j$. We need to find a base-point-preserving 1-Lipschitz $u: Y \rightarrow X_{\infty}$ such that $\pi_{i}^{\infty} \circ u=u_{i}$ for all $i \in I$. For $y \in Y$ set $u(y):=\left(u_{i}(y)\right)_{i \in I} \in \prod_{i \in I} X_{i}$. Since each $u_{i}$ is base-point-preserving, $u(\star)=\left(\star_{i}\right)_{i \in I}=\star_{\infty}$. Since each $u_{i}$ is 1-Lipschitz,

$$
\sup _{i \in I} d_{i}\left(\star_{i}, u_{i}(y)\right)=\sup _{i \in I} d_{i}\left(u_{i}(\star), u_{i}(y)\right) \leq d_{Y}(\star, y)<\infty
$$

This shows that $u$ is a base-point-preserving map into $X_{\infty}$. Moreover, the function $u$ is 1-Lipschitz for the same reason: given $y, y^{\prime} \in Y$, then

$$
d_{\infty}\left(u(y), u\left(y^{\prime}\right)\right)=\sup _{i \in I} d_{i}\left(u_{i}(y), u_{i}\left(y^{\prime}\right)\right) \leq d_{Y}\left(y, y^{\prime}\right)
$$

Hence $u: Y \rightarrow X_{\infty}$ is a base-point-preserving 1-Lipschitz map. It is clear that $u: Y \rightarrow X$ is the only map that satisfies $\pi_{i}^{\infty} \circ u=u_{i}$ for all $i \in I$.

It is interesting to note that $\left(X_{\infty}, \star_{\infty}\right)$ is not the inverse limit in the category of pointed metric spaces with base-point-preserving submetries. Indeed, in this category inverse limits do not exist in general as the following example demonstrates. This does not come as a surprise because submetries being surjective are strongly restricted.

Example 2.4. For each $n \in \mathbb{N}$ let $X_{n}$ be the metric space with $n$ elements denoted by $\left\{a_{1}, \ldots, a_{n}\right\}$ and pairwise distances 1 for different points. The projection $\pi_{n+1}: X_{n+1} \rightarrow X_{n}$ is given by $\pi_{n+1}\left(a_{i}\right)=a_{i}$ for $i \leq n$ 
and $\pi_{n+1}\left(a_{n+1}\right)=a_{n}$. These maps are clearly submetries. Let $Y=\left\{a_{i}, b_{i}\right\}_{i \in \mathbb{N}} \cup\{b\}$ be the countable metric space with distances $d\left(a_{i}, a_{j}\right)=d\left(b_{i}, b_{j}\right)=d\left(b_{i}, b\right)=1$ for $i \neq j$ and $d\left(a_{i}, b_{j}\right)=d\left(a_{i}, b\right)=2$ for all $i, j \in \mathbb{N}$. Let $u_{n}: Y \rightarrow X_{n}$ be the map given by $u_{n}\left(a_{i}\right)=u_{n}\left(b_{i}\right)=a_{i}$ for $i \leq n$ and $u_{n}(y)=a_{n}$ for all other points $y \in Y$. These maps $u_{n}$ are compatible with the projections $\pi_{n}$ and submetries. For the latter note that different fibers in $Y$ have distance $\operatorname{dist}\left(u_{n}^{-1}\left(a_{i}\right), u_{n}^{-1}\left(a_{j}\right)\right)=1=d_{n}\left(a_{i}, a_{j}\right)$ and this distance is realized for any point in one of the fibers.

Now consider any other metric space $X$ with submetries $\pi_{n}^{\infty}: X \rightarrow X_{n}$ with the compatibility condition $\pi_{n}^{n+1}$ 。 $\pi_{n+1}^{\infty}=\pi_{n}^{\infty}$. We show that the universal property that would identify $X$ as the inverse limit of $\left(\left(X_{n}\right)_{n \in \mathbb{N}},\left(\pi_{n}\right)_{n \in \mathbb{N}}\right)$ in the category of metric spaces with submetries does not hold. So assume that there is submetry $u: Y \rightarrow X$ with $u_{n}=\pi_{n}^{\infty} \circ u$ for all $n \in \mathbb{N}$. First note that $u^{-1}(u(b))=\{b\}$ because for any other point $y \neq b$ in $Y$ there exists some $n \geq 1$ such that $u_{n}(y) \neq u_{n}(b)$. For the same reason, the fiber $u^{-1}\left(u\left(a_{i}\right)\right)$ is either $\left\{a_{i}\right\}$ or $\left\{a_{i}, b_{i}\right\}$. Assume first that there exists some $i$ such that $u^{-1}\left(u\left(a_{i}\right)\right)=\left\{a_{i}, b_{i}\right\}$. Then

$$
\operatorname{dist}\left(u^{-1}\left(u\left(a_{i}\right)\right), u^{-1}(u(b))\right)=\operatorname{dist}\left(\left\{a_{i}, b_{i}\right\},\{b\}\right)=1
$$

but this distance is not realized by the point $a_{i}$ in the first fiber. This is not possible and thus $u^{-1}\left(u\left(a_{i}\right)\right)=\left\{a_{i}\right\}$ for all $i$ and hence also $u^{-1}\left(u\left(b_{i}\right)\right)=\left\{b_{i}\right\}$ for all $i$. This implies that $u: Y \rightarrow X$ as an injective submetry is an isometry.

Let $Y^{\prime}$ be the metric space obtained from $Y$ by changing all the distances with value 2 to $3 / 2$. In this case too, all the projections $u_{n}: Y^{\prime} \rightarrow X_{n}$ are submetries. The same reasoning as above implies that any compatible submetry $Y^{\prime} \rightarrow X$ has to be an isometry. But $Y$ and $Y^{\prime}$ are obviously not isometric. This shows that $\left(X,\left(\pi_{n}^{\infty}\right)_{n \in \mathbb{N}}\right)$ is not the inverse limit of $\left(\left(X_{n}\right)_{n \in \mathbb{N}},\left(\pi_{n}\right)_{n \in \mathbb{N}}\right)$ and thus there is in general no inverse limit in the category of metric spaces with submetries as morphisms.

In what follows we introduce additional properties for maps. We recall that a rectifiable path $\gamma:[a, b] \rightarrow X$ in a metric space $X$ is a continuous map with finite length. The precise definition and relevant properties of the length functional can be found for example in [3].

Definition 2.5. A submetry $\pi: X \rightarrow Y$ has the unique path lifting property if for all rectifiable paths $\gamma$ : $[a, b] \rightarrow Y$ and each point $x \in \pi^{-1}(\gamma(a))$ there is path $\bar{\gamma}:[a, b] \rightarrow X$ such that

(1) $L(\bar{\gamma})<\infty$,

(2) $\bar{\gamma}(a)=x$,

(3) $\pi(\bar{\gamma}(t))=\gamma(t)$ for all $t \in[a, b]$,

(4) if a path $\eta:[a, b] \rightarrow X$ satisfies (1),(2) and (3), then $\eta=\bar{\gamma}$,

(5) $L(\bar{\gamma}) \leq L(\gamma)$ (because $\pi$ is 1-Lipschitz we have equality).

Such a path $\bar{\gamma}$ is called a lift of $\gamma$.

Lemma 2.6. Assume that $\pi: X \rightarrow Y$ has the unique path lifting property and $\bar{\gamma}$ is a lift of a rectifiable path $\gamma:[a, b] \rightarrow Y$. The following hold:

(a) If $a \leq s<t \leq b$, then $L\left(\left.\bar{\gamma}\right|_{[s, t]}\right)=L\left(\left.\gamma\right|_{[s, t]}\right)$.

(b) If $\gamma$ is $L$-Lipschitz, then also $\bar{\gamma}$ is $L$-Lipschitz.

(c) If $\eta:[a, b] \rightarrow X$ is rectifiable, then $\eta$ is the unique lift of $\pi \circ \eta$ starting at $\eta(a)$.

Proof. (a): The path $\left.\bar{\gamma}\right|_{[s, t]}$ satisfies (1), (2) and (3) in Definition 2.5 for the interval $[s, t] \subset[a, b]$ and the starting point $\bar{\gamma}(s)$. Hence $\left.\bar{\gamma}\right|_{[s, t]}$ is the unique lift with these properties by (4) and thus $L\left(\left.\bar{\gamma}\right|_{[s, t]}\right)=L\left(\left.\gamma\right|_{[s, t]}\right)$ by $(5)$. Alternatively, (a) also follows directly from $\operatorname{Lip}(\pi) \leq 1$ and $L(\bar{\gamma})=L(\gamma)$.

(b): If moreover $\gamma$ is $L$-Lipschitz, then

$$
d_{X}(\bar{\gamma}(s), \bar{\gamma}(t)) \leq L\left(\left.\bar{\gamma}\right|_{[s, t]}\right) \leq L\left(\left.\gamma\right|_{[s, t]}\right) \leq L|t-s|
$$


Hence also $\bar{\gamma}$ is $L$-Lipschitz.

(c): Since $\pi$ is Lipschitz, also the projection $\pi \circ \bar{\gamma}$ is rectifiable. It is clear that $\bar{\gamma}$ is a lift of $\pi \circ \bar{\gamma}$ and the uniqueness follows from the assumption on $\pi: X \rightarrow Y$.

Next we show that the unique path lifting property is inherited by $X_{\infty}$.

Lemma 2.7. Assume that $\pi_{i+1}:\left(X_{i+1}, \star_{i+1}\right) \rightarrow\left(X_{i}, \star_{i}\right)$ is base-point-preserving and has the unique path lifting property for every $i \in \mathbb{N}$. Then $\pi_{i}^{\infty}: X_{\infty} \rightarrow X_{i}$ also has the unique path lifting property for every $i \in \mathbb{N}$.

Proof. From Lemma 2.2 we already know that each $\pi_{i}^{\infty}$ is a submetry. Fix some $j \in \mathbb{N}$ and let $\gamma_{j}:[a, b] \rightarrow X_{j}$ be a rectifiable path and let $x=\left(x_{1}, x_{2}, \ldots\right) \in X_{\infty}$ be a point with $x_{j}=\gamma_{j}(a)$. Such a point exists because $\pi_{j}^{\infty}$ as a submetry is surjective. For $i<j$, let $\gamma_{i}:=\pi_{i}^{j} \circ \gamma_{j}$. For $i>j$ let $\gamma_{i}$ be the successive lift of $\gamma_{j}$ with $\gamma_{i}(a)=x_{i}$. First we show that $\gamma_{\infty}(t):=\left(\gamma_{1}(t), \gamma_{2}(t), \ldots\right)$ is in $X_{\infty}$ for each $t \in[a, b]$. Because lifts are length-preserving,

$$
d_{i}\left(x_{i}, \gamma_{i}(t)\right) \leq L\left(\gamma_{i}\right) \leq L\left(\gamma_{j}\right)
$$

for all $i>j$ and the same holds true for $i<j$ because each $\pi_{i}$ is 1-Lipschitz. Hence

$$
\sup _{i \geq 1} d_{i}\left(\star_{i}, \gamma_{i}(t)\right) \leq \sup _{i \geq 1} d_{i}\left(\star_{i}, x_{i}\right)+\sup _{i \geq 1} d_{i}\left(x_{i}, \gamma_{i}(t)\right)<\infty,
$$

which implies that $\gamma_{\infty}(t) \in X_{\infty}$ for all $t \in[a, b]$. From Lemma 2.6 it follows that $L\left(\left.\gamma_{i}\right|_{[s, t]}\right)=L\left(\left.\gamma_{j}\right|_{[s, t]}\right)$ for all $a \leq s<t \leq b$ and $i \in \mathbb{N}$. Further

$$
\begin{aligned}
d_{\infty}\left(\gamma_{\infty}(s), \gamma_{\infty}(t)\right) & =\sup _{i \geq 1} d_{i}\left(\gamma_{i}(s), \gamma_{i}(t)\right) \leq \sup _{i \geq 1} L\left(\left.\gamma_{i}\right|_{[s, t]}\right) \\
& \leq L\left(\left.\gamma_{j}\right|_{[s, t]}\right)
\end{aligned}
$$

This shows that $\gamma_{\infty}$ is continuous since $L\left(\left.\gamma_{j}\right|_{[s, t]}\right) \rightarrow 0$ for $t-s \rightarrow 0$. By taking a finite partition of $[a, b]$ and summing up the distances it is immediate that $L\left(\gamma_{\infty}\right) \leq L\left(\gamma_{j}\right)$. So $\gamma_{\infty}$ is a lift of $\gamma_{j}$ and it remains to show the uniqueness of $\gamma_{\infty}$.

Assume that $\eta:[a, b] \rightarrow X_{\infty}$ is a rectifiable path with $\pi_{j}^{\infty}(\eta(t))=\gamma_{j}(t)$ for all $t$ as well as $\eta(a)=x$. Then for each $i>j$, the path $\pi_{i}^{\infty} \circ \eta$ is a lift of $\gamma_{j}$ with $\pi_{i}^{\infty}(\eta(a))=x_{i}$. By the unique path lifting property it follows that $\pi_{i}^{\infty}(\eta(t))=\pi_{i}^{\infty}\left(\gamma_{\infty}(t)\right)$ for all $t$ and $i$. Hence $\eta=\gamma_{\infty}$ and we are done.

It follows from Lemma 2.6(c) that $\gamma_{\infty}:[a, b] \rightarrow X_{\infty}$ is uniquely defined by $\gamma_{\infty}(a)$ and $\pi_{i}^{\infty} \circ \gamma_{\infty}$ for any given $i$. We call such a rectifiable path $\gamma_{\infty}$ the lift of its projections $\pi_{i}^{\infty} \circ \gamma_{\infty}$ at the starting point $\gamma_{\infty}(a)$.

Definition 2.8. A map $\pi: X \rightarrow Y$ has the continuous path lifting property if it has the unique path lifting property and for each sequence of paths $\gamma_{n}:[0,1] \rightarrow Y, n \in \mathbb{N}$, with

(1) $\gamma_{n}(0)=y$ for all $n$,

(2) $\sup _{n \geq 1} \operatorname{Lip}\left(\gamma_{n}\right)<\infty$

(3) $\gamma(t) \stackrel{=}{=} \lim _{n \rightarrow \infty} \gamma_{n}(t)$ for all $t \in[0,1]$,

it holds that

$$
\bar{\gamma}(t)=\lim _{n \rightarrow \infty} \bar{\gamma}_{n}(t) \quad \text { for all } \quad t \in[0,1]
$$

where $\bar{\gamma}_{n}$ and $\bar{\gamma}$ are lifts of $\gamma_{n}$ and $\gamma$, respectively, with the same starting point $\bar{\gamma}_{n}(0)=\bar{\gamma}(0) \in \pi^{-1}(y)$.

Note that in the definition above, assuming uniform bounds on Lipschitz constants, pointwise convergence and uniform convergence are equivalent. 
Recall that a metric space $(X, d)$ is proper if all the closed balls $\mathbf{B}(x, r)$ in $X$ are compact. The metric space $(X, d)$ is a geodesic space if for all points $x, y \in X$ there is a path $\gamma:[a, b] \rightarrow X$ with $\gamma(a)=x, \gamma(b)=y$ and $L(\gamma)=d(x, y)$. Any such path is called a geodesic. A path $\gamma:[a, b] \rightarrow X$ is parametrized proportionally to arc length if $L\left(\left.\gamma\right|_{[s, t]}\right)|b-a|=L(\gamma)|t-s|$ for all $a \leq s \leq t \leq b$. It is a general fact in metric geometry that any rectifiable path $\gamma$ has a reparametrization $\tilde{\gamma} \circ \phi$ by arc length, i.e., $L\left(\left.\tilde{\gamma}\right|_{[s, t]}\right)=|t-s|$ for all $s$ and $t$.

Proposition 2.9. Assume that for every $i \in \mathbb{N}$, the pointed metric space $\left(X_{i}, \star_{i}\right)$ is geodesic and proper. If all the projections $\pi_{i+1}: X_{i+1} \rightarrow X_{i}$ are base-point-preserving and have the continuous path lifting property, then $X_{\infty}$ is a complete geodesic metric space and for each pair of points $x=\left(x_{1}, x_{2}, \ldots\right), y=\left(y_{1}, y_{2}, \ldots\right) \in X_{\infty}$ there exist $\eta_{\infty}:[0,1] \rightarrow X_{\infty}$ and $\gamma^{\sigma(n)}:[0,1] \rightarrow X_{\sigma(n)}$ for a strictly increasing sequence $\sigma: \mathbb{N} \rightarrow \mathbb{N}$ such that:

(A) $\eta_{\infty}$ is a geodesic parametrized proportionally to arc length connecting $x$ with $y$ in $X_{\infty}$.

(B) Each $\gamma^{\sigma(n)}$ is a geodesic parametrized proportionally to arc length connecting $x_{\sigma(n)}$ with $y_{\sigma(n)}$,

(C) $\pi_{i}^{\infty} \circ \eta_{\infty}=\lim _{n \rightarrow \infty} \pi_{i}^{\sigma(n)} \circ \gamma^{\sigma(n)}$ for all $i \in \mathbb{N}$.

Proof. Note that proper metric spaces are in particular complete, so it already follows from Lemma 2.2 that $X_{\infty}$ is a complete metric space. For each $n \in \mathbb{N}$ let $\gamma^{n}:[0,1] \rightarrow X_{k}$ be a geodesic parametrized proportionally to arc length connecting $x_{n}$ with $y_{n}$. This implies that $\operatorname{Lip}\left(\gamma^{n}\right) \leq d_{n}\left(x_{n}, y_{n}\right)$ for all $n \in \mathbb{N}$. From Lemma 2.7 it follows that the lift $\gamma_{\infty}^{n}:[0,1] \rightarrow X_{\infty}$ of $\gamma^{n}$ with $\gamma_{\infty}^{n}(0)=x$ satisfies

$$
\operatorname{Lip}\left(\gamma_{\infty}^{n}\right) \leq \operatorname{Lip}\left(\gamma^{n}\right) \leq d_{n}\left(x_{n}, y_{n}\right) \leq d_{\infty}(x, y)
$$

Hence the path $\gamma_{\infty}^{n}$ is contained in the closed ball $\mathbf{B}\left(x, d_{\infty}(x, y)\right)$. Since each $\pi_{i}^{\infty}$ is 1-Lipschitz by Lemma 2.2, the path $\pi_{i}^{\infty} \circ \gamma_{\infty}^{n}$ is contained in $\mathbf{B}\left(x_{i}, d_{\infty}(x, y)\right)$, starts at $x_{i}$ and satisfies $\operatorname{Lip}\left(\pi_{i}^{\infty} \circ \gamma_{\infty}^{n}\right) \leq d_{\infty}(x, y)$ for all $i$ and $n$. Since $\mathbf{B}\left(x_{1}, d_{\infty}(x, y)\right)$ is compact, the theorem of Arzelà-Ascoli guarantees a subsequence $(\sigma(1, n))_{n \in \mathbb{N}}$ of $(n)_{n \in \mathbb{N}}$ such that $\left(\pi_{1}^{\infty} \circ \gamma_{\infty}^{\sigma(1, n)}\right)_{n \in \mathbb{N}}$ converges uniformly to some path $\eta_{1}$. Recursively we find for every $i \geq 2$ a subsequence $(\sigma(i, n))_{n \in \mathbb{N}}$ of $(\sigma(i-1, n))_{n \in \mathbb{N}}$ such that $\left(\pi_{i}^{\infty} \circ \gamma_{\infty}^{\sigma(i, n)}\right)_{n \in \mathbb{N}}$ converges uniformly to some path $\eta_{i}$. For the diagonal sequence it holds that

$$
\lim _{n \rightarrow \infty} \pi_{i}^{\infty} \circ \gamma_{\infty}^{\sigma(n, n)}=\eta_{i}
$$

uniformly for all $i \in \mathbb{N}$. We have the following properties of these limits for all $i \in \mathbb{N}$ :

(1) $\eta_{i}(0)=x_{i}$

(2) $\eta_{i}(1)=y_{i}$

(3) $\operatorname{Lip}\left(\eta_{i}\right) \leq d_{\infty}(x, y)$,

(4) $\pi_{i+1}\left(\eta_{i+1}\right)=\eta_{i}$.

(1) is clear by construction since $\pi_{i}^{\infty} \circ \gamma_{\infty}^{n}(0)=x_{i}$ for all $i, n \in \mathbb{N}$. Similarly, (2) follows from the fact that $\pi_{i}^{\infty} \circ \gamma_{\infty}^{n}(1)=y_{i}$ for all $i \leq n$. (3) is a consequence of $\operatorname{Lip}\left(\pi_{i}^{\infty} \circ \gamma_{\infty}^{n}\right) \leq d_{\infty}(x, y)$ for all $i, n \in \mathbb{N}$. To see (4) note that the path $\pi_{i+1}^{\infty} \circ \gamma_{\infty}^{\sigma(n, n)}$ is the unique lift of $\pi_{i}^{\infty} \circ \gamma_{\infty}^{\sigma(n, n)}$ starting at $x_{i+1}$ for all $i, n \in \mathbb{N}$. The continuous path lifting property now implies that $\eta_{i+1}$ is the unique lift of $\eta_{i}$ for each $i \in \mathbb{N}$. Hence (4) holds.

It now follows from $(1),(3),(4)$ and Lemma 2.7 that $\eta_{\infty}(t)=\left(\eta_{1}(t), \eta_{2}(t), \ldots\right)$ is for each $i \in \mathbb{N}$ the unique lift of $\eta_{i}$ starting at $x$. Moreover $\operatorname{Lip}\left(\eta_{\infty}\right) \leq d_{\infty}(x, y)$ by $(3)$ and Lemma 2.6. The equality $\eta_{\infty}(1)=y$ holds because of $(2)$ and hence $\eta_{\infty}$ is a geodesic connecting $x$ with $y$. The bound $\operatorname{Lip}\left(\eta_{\infty}\right) \leq d_{\infty}(x, y)$ also implies that $\eta_{\infty}$ is parametrized proportionally to arc length.

Note that in the setting of the proposition above the projections $\pi_{i}^{\infty}: X_{\infty} \rightarrow X_{i}$ in general do not have the continuous path lifting property as we will demonstrate in Remark 4.6 below. 


\section{Group structures AND their Limits}

We consider the case where each metric space $X_{i}$ has a group structure with a left invariant distance. The following lemma shows that then also the metric space $X_{\infty}$ admits a group structure making the distance left invariant.

Lemma 3.1. Assume that $\left(X_{i}, e_{i}\right)_{i \in I}$ is a collection of pointed metric groups together with maps $\pi_{j}^{i}: X_{i} \rightarrow X_{j}$ for $i \geq j$ such that:

(A) $e_{i}$ is the identity element of $X_{i}$,

(B) the projections $\pi_{j}^{i}: X_{i} \rightarrow X_{j}$ are homomorphisms,

(C) left translations are isometries, i.e., for each $g_{i}, x_{i}, y_{i} \in X_{i}$ it holds that

$$
d_{i}\left(g_{i} \cdot x_{i}, g_{i} \cdot y_{i}\right)=d_{i}\left(x_{i}, y_{i}\right)
$$

Then the limit $\left(X_{\infty}, e_{\infty}\right)$ is a group with identity element $e_{\infty}=\left(e_{i}\right)_{i \in I}$, and operations $\left(x_{i}\right)_{i \in I}^{-1}:=\left(x_{i}^{-1}\right)_{i \in I}$ as well as $\left(x_{i}\right)_{i \in I} \cdot\left(y_{i}\right)_{i \in I}:=\left(x_{i} \cdot y_{i}\right)_{i \in I}$. Moreover, left translations in $X_{\infty}$ are isometries with respect to $d_{\infty}$.

Proof. We need to show that

(1) $e_{\infty} \in X_{\infty}$,

(2) $x^{-1} \in X_{\infty}$ if $x \in X_{\infty}$,

(3) $x \cdot y \in X_{\infty}$ if $x, y \in X_{\infty}$,

(4) $d_{\infty}(g \cdot x, g \cdot y)=d_{\infty}(x, y)$ for all $g, x, y \in X_{\infty}$.

(1) is clear by definition. To see (2) let $x=\left(x_{i}\right)_{i \in I} \in X_{\infty}$. By assumption it holds that

$$
\sup _{i \in I} d_{i}\left(e_{i}, x_{i}^{-1}\right)=\sup _{i \in I} d_{i}\left(x_{i} \cdot e_{i}, x_{i} \cdot x_{i}^{-1} \cdot e_{i}\right)=\sup _{i \in I} d_{i}\left(x_{i}, e_{i}\right)<\infty
$$

Because each $\pi_{j}^{i}$ is a homomorphism $\pi_{j}^{i}\left(x_{i}^{-1}\right)=\pi_{j}^{i}\left(x_{i}\right)^{-1}=x_{i}^{-1}$ and this implies that $\left(x_{i}\right)_{i \in I}^{-1} \in X_{\infty}$. For (3) note that by left invariance of each $d_{i}$ and by (2) it holds

$$
\sup _{i \in I} d_{i}\left(e_{i}, x_{i} \cdot y_{i}\right)=\sup _{i \in I} d_{i}\left(x_{i}^{-1}, y_{i}\right) \leq \sup _{i \in I} d_{i}\left(x_{i}^{-1}, e_{i}\right)+d_{i}\left(e_{i}, y_{i}\right)<\infty
$$

Moreover, $\pi_{j}^{i}\left(x_{i} \cdot y_{i}\right)=\pi_{j}^{i}\left(x_{i}\right) \cdot \pi_{j}^{i}\left(y_{i}\right)=x_{j} \cdot y_{j}$ again because all $\pi_{j}^{i}$ are homomorphisms. Thus $\left(x_{i} \cdot y_{i}\right)_{i \in I} \in X_{\infty}$. Clearly,

$$
\begin{aligned}
d_{\infty}(g \cdot x, g \cdot y) & =\sup _{i \in I} d_{i}\left(g_{i} \cdot x_{i}, g_{i} \cdot y_{i}\right)=\sup _{i \in I} d_{i}\left(x_{i}, y_{i}\right) \\
& =d_{\infty}(x, y)
\end{aligned}
$$

and this shows (4).

We will see later in Theorem 5.1 that right translations in $X_{\infty}$ are in general not continuous, and the resulting limit space thus is not a topological group. A particular example of this behaviour is given by the sequence $X_{i}=G^{i}\left(\mathbb{R}^{n}\right)$ of free nilpotent Lie groups of step $i$ over $\mathbb{R}^{n}$ equipped with the Carnot-Carathéodory metric. 


\section{SPACE OF SIgNATURES AS AN INVERSE Limit}

We first review the basic definitions and results about signatures and free nilpotent Lie groups, we mainly refer to [7]. As defined in Definition 7.2 of [7] the step $\mathbf{k}$ signature of a rectifiable path $\gamma:[a, b] \rightarrow \mathbb{R}^{n}$ is given by

$$
\begin{aligned}
S_{k}(\gamma)_{a, b} & :=\left(1, \int_{a<t<b} \mathrm{~d} \gamma_{t}, \ldots, \int_{a<t_{1}<\cdots<t_{k}<b} \mathrm{~d} \gamma_{t_{1}} \otimes \cdots \otimes \mathrm{d} \gamma_{t_{k}}\right) \\
& \in T^{k}\left(\mathbb{R}^{n}\right):=\prod_{i=0}^{k}\left(\mathbb{R}^{n}\right)^{\otimes i}
\end{aligned}
$$

Note that the iterated integrals are interpreted as Riemann-Stieltjes integrals and thus well defined and independent of the particular parametrization of $\gamma$. There are natural coordinate projections $\xi_{k}: T^{l}\left(\mathbb{R}^{n}\right) \rightarrow\left(\mathbb{R}^{n}\right)^{\otimes k}$ and $\pi_{k}^{l}: T^{l}\left(\mathbb{R}^{n}\right) \rightarrow T^{k}\left(\mathbb{R}^{n}\right)$ for $0 \leq k \leq l$. For $g, h \in T^{k}\left(\mathbb{R}^{n}\right)$ the truncated tensor product is defined by

$$
g \otimes_{k} h:=\sum_{0 \leq i+j \leq k} \xi_{i}(g) \otimes \xi_{j}(h) \in T^{k}\left(\mathbb{R}^{n}\right)
$$

which makes $\left(T^{k}\left(\mathbb{R}^{n}\right),+, \otimes_{k}\right)$ into an associative algebra with neutral element $1_{k}:=(1,0, \ldots, 0)$, see Proposition 7.4 of [7]. Similarly one defines the infinite tensor algebra $T^{\infty}\left(\mathbb{R}^{n}\right):=\prod_{i=0}^{\infty}\left(\mathbb{R}^{n}\right)^{\otimes i}$.

Given a rectifiable path $\gamma:[a, b] \rightarrow \mathbb{R}^{n}$, Chen's theorem states that for $a \leq s<t<u \leq b$

$$
S_{k}(\gamma)_{s, u}=S_{k}(\gamma)_{s, t} \otimes_{k} S_{k}(\gamma)_{t, u}
$$

see e.g. Theorem 7.11 of [7].

Set $\mathfrak{t}^{k}\left(\mathbb{R}^{n}\right):=\left\{g \in T^{k}\left(\mathbb{R}^{n}\right): \xi_{0}(g)=0\right\}$. It is shown in Proposition 7.17 of [7] that the set $1_{k}+\mathfrak{t}^{k}\left(\mathbb{R}^{n}\right) \subset$ $T^{k}\left(\mathbb{R}^{n}\right)$ together with the truncated tensor product $\otimes_{k}$ of $T^{k}\left(\mathbb{R}^{n}\right)$ is a Lie group. Similarly, the set $\mathfrak{t}^{k}\left(\mathbb{R}^{n}\right) \subset$ $T^{k}\left(\mathbb{R}^{n}\right)$ together with the bilinear map

$$
[g, h]:=g \otimes_{k} h-h \otimes_{k} g
$$

is a Lie algebra, Proposition 7.19 of $[7]$. By $\mathfrak{g}^{k}\left(\mathbb{R}^{n}\right) \subset \mathfrak{t}^{k}\left(\mathbb{R}^{n}\right)$ we denote the smallest Lie subalgebra that contains $\left(\mathbb{R}^{n}\right)^{\otimes 1} \subset \mathfrak{t}^{k}\left(\mathbb{R}^{n}\right)$. The exponential map $\exp _{k}: \mathfrak{t}^{k}\left(\mathbb{R}^{n}\right) \rightarrow 1_{k}+\mathfrak{t}^{k}\left(\mathbb{R}^{n}\right), \exp _{k}(a):=1_{k}+\sum_{i=1}^{k} \frac{a^{\otimes i}}{i !}, \operatorname{maps} \mathfrak{g}^{k}\left(\mathbb{R}^{n}\right)$ onto a closed Lie subgroup $G^{k}\left(\mathbb{R}^{n}\right):=\exp _{k}\left(\mathfrak{g}^{k}\left(\mathbb{R}^{n}\right)\right)$ of $1_{k}+\mathfrak{t}^{k}$, the free nilpotent Lie group of step $\mathbf{k}$ over $\mathbb{R}^{n}$, Corollary 7.27 of [7]. The straight path $\gamma:[0,1] \rightarrow \mathbb{R}^{n}$ given by $\gamma(t)=t v$ for some $v \in \mathbb{R}^{n}$ has truncated signature $S_{k}(\gamma)_{0,1}=\exp _{k}(v)$, Example 7.21 of [7]. Chow's theorem states that for any $g \in G^{k}\left(\mathbb{R}^{n}\right)$ there exists a piecewise linear path $\gamma:[0,1] \rightarrow \mathbb{R}^{n}$ such that $g=S_{k}(\gamma)_{0,1}$, see e.g. Theorem 7.28 of [7]. Moreover,

$$
G^{k}\left(\mathbb{R}^{n}\right)=\left\{S_{k}(\gamma)_{0,1}: \gamma:[0,1] \rightarrow \mathbb{R}^{n} \text { is rectifiable }\right\},
$$

Theorem 7.30 of [7]. The Carnot-Carathéodory norm on $G^{k}\left(\mathbb{R}^{n}\right)$ is defined by

$$
\|g\|:=\inf \left\{L(\gamma): \gamma:[0,1] \rightarrow \mathbb{R}^{n} \text { is rectifiable and } S_{k}(\gamma)_{0,1}=g\right\} .
$$

This infimum is achieved by some rectifiable path $\gamma$, see Theorem 7.32 of [7], and the Carnot-Carathéodory metric $d_{k}(g, h):=\left\|g^{-1} \otimes_{k} h\right\|$ is a left invariant geodesic metric on $G^{k}\left(\mathbb{R}^{n}\right)$, see e.g. Definition 7.41 of [7]. By construction it is clear that $\pi_{k}^{l}: G^{l}\left(\mathbb{R}^{n}\right) \rightarrow G^{k}\left(\mathbb{R}^{n}\right)$ is 1-Lipschitz for any $1 \leq k \leq l$. 
Let $\gamma:[a, b] \rightarrow \mathbb{R}^{n} \cong G^{1}\left(\mathbb{R}^{n}\right)$ be a rectifiable path and let $g \in G^{k}\left(\mathbb{R}^{n}\right)$ with $\pi_{1}^{k}(g)=\gamma(a)$. We define $\gamma_{k}$ : $[a, b] \rightarrow G^{k}\left(\mathbb{R}^{n}\right)$ by

$$
\gamma_{k}(t):=g \otimes_{k} S_{k}(\gamma)_{a, t},
$$

for $t \in[a, b]$. Clearly $\pi_{1}^{k} \circ \gamma_{k}=\gamma, \gamma_{k}(0)=g$ and by Proposition 7.59 of [7] and the left invariance of $d_{k}$ we obtain that

$$
L\left(\gamma_{k}\right)=L\left(t \mapsto S_{k}(\gamma)_{a, t}\right)=L(\gamma)
$$

This implies that all the projections

$$
\pi_{k+1}:\left(G^{k+1}\left(\mathbb{R}^{n}\right), d_{k+1}, 1_{k+1}\right) \rightarrow\left(G^{k}\left(\mathbb{R}^{n}\right), d_{k}, 1_{k}\right)
$$

have the unique path lifting property as in Definition 2.5. The fact that these projections also have the continuous path lifting property, in the sense of Definition 2.8, is certainly known but for the sake of convenience we add a proof here.

Lemma 4.1. For every $k \in \mathbb{N}$, the projection

$$
\pi_{k+1}:\left(G^{k+1}\left(\mathbb{R}^{n}\right), d_{k+1}, 1_{k+1}\right) \rightarrow\left(G^{k}\left(\mathbb{R}^{n}\right), d_{k}, 1_{k}\right)
$$

has the continuous path lifting property. Moreover, each each metric space $G^{k}\left(\mathbb{R}^{n}\right)$ is geodesic and proper.

Proof. The standard topology on $1_{k}+\mathfrak{t}^{k}\left(\mathbb{R}^{n}\right) \subset T^{k}\left(\mathbb{R}^{n}\right)=\prod_{i=0}^{k}\left(\mathbb{R}^{n}\right)^{\otimes i}$ is induced by the distance function

$$
\rho(x, y):=\max _{i=1, \ldots, k}\left|\xi_{i}(x)-\xi_{i}(y)\right| .
$$

In fact, as shown in Proposition 7.45 of [7], there is a constant $C \geq 1$ such that

$$
C^{-1} \rho\left(1_{k}, g\right) \leq \max \left\{d_{k}\left(1_{k}, g\right), d_{k}\left(1_{k}, g\right)^{k}\right\} \leq C \rho\left(1_{k}, g\right)
$$

for all $g \in G^{k}\left(\mathbb{R}^{n}\right)$. Thus the topology of $G^{k}\left(\mathbb{R}^{n}\right)$ agrees with the topology induced by $\rho$ and since closed balls in $G^{k}\left(\mathbb{R}^{n}\right)$ are compact each $G^{k}\left(\mathbb{R}^{n}\right)$ proper. We already stated above that $G^{k}\left(\mathbb{R}^{n}\right)$ is geodesic.

If $f, g:[0,1] \rightarrow \mathbb{R}$ are Lipschitz, then we claim that also the function $\iota(t):=\int_{0}^{t} f \mathrm{~d} g$ is Lipschitz. Indeed, for $0 \leq s \leq t \leq 1$ it holds that

$$
\begin{aligned}
|\iota(s)-\iota(t)| & =\left|\int_{s}^{t} f(t) g^{\prime}(t) \mathrm{d} t\right| \leq \int_{s}^{t}\left|f(t) g^{\prime}(t)\right| \mathrm{d} t \\
& \leq\|f\|_{\infty} \operatorname{Lip}(g)|t-s| \\
& \leq(f(0)+\operatorname{Lip}(f)) \operatorname{Lip}(g)|t-s| .
\end{aligned}
$$

Moreover, we claim that if $\left(f_{m}\right)_{m \in \mathbb{N}}$ and $\left(g_{m}\right)_{m \in \mathbb{N}}$ are sequences of Lipschitz functions on $[0,1]$ with $\sup _{m}\left\{\operatorname{Lip}\left(f_{m}\right), \operatorname{Lip}\left(g_{m}\right)\right\}<\infty$ that converge pointwise (and thus uniformly) to $f$ and $g$, respectively, then

$$
\lim _{m \rightarrow \infty} \int_{0}^{1} f_{m} \mathrm{~d} g_{m}=\int_{0}^{1} f \mathrm{~d} g
$$

This follows for example by properties of the Riemann-Stieltjes integral, see [12] for more general convergence results where the statement above is attributed to Helly [9]. Alternatively, one has another proof of (4.3) by 
observing that the characteristic function $\chi_{[0,1]}$ induces a 1-dimensional metric current on $\mathbb{R}$, see Example 3.2 of $[1]$.

Assume now that $\gamma_{m}:[0,1] \rightarrow \mathbb{R}^{n}, m \in \mathbb{N}$, is a sequence of Lipschitz paths with $\gamma_{m}(0)=0, \sup _{m} \operatorname{Lip}\left(\gamma_{m}\right)<$ $\infty$, and $\lim _{m \rightarrow \infty}\left\|\gamma_{m}-\gamma\right\|_{\infty}=0$. It follows by induction on $k$ using (4.2) and (4.3) that

$$
\lim _{m \rightarrow \infty} \rho\left(S_{k}\left(\gamma_{m}\right)_{0, t}, S_{k}(\gamma)_{0, t}\right)=0
$$

for all $t \in[0,1]$. By the discussion in the first part of the proof this implies that

$$
\lim _{m \rightarrow \infty} d_{k}\left(S_{k}\left(\gamma_{m}\right)_{0, t}, S_{k}(\gamma)_{0, t}\right)=0
$$

The general continuous lifting property now follows from the left invariance of the metric and the unique path lifting property for the projections $\pi_{1}^{k}: G^{k}\left(\mathbb{R}^{n}\right) \rightarrow G^{1}\left(\mathbb{R}^{n}\right) \cong \mathbb{R}^{n}$.

For the discussions that follow, metric trees play a fundamental role. Let us recall the following definitions. An arc in a topological space $X$ is a set $I \subset X$ homeomorphic to [0,1]. By abuse of notation we denote by $\partial I$ the end points of $I$. The space $X$ is said to be arcwise connected if for any two different points $x, y \in X$ there is an arc connecting $x$ with $y$, i.e., there is an arc $I \subset X$ such that $\partial I=\{x, y\}$.

A topological tree is a metrizable topological space $X$ with the following two properties:

(1) $X$ is uniquely arcwise connected, i.e., for any two different points $x, y \in X$ there is a unique $\operatorname{arc}$ in $X$ connecting $x$ with $y$. We will denote this arc by $[x, y]$.

(2) $X$ is locally arcwise connected, i.e., for any $x \in X$ and any neighbourhood $U$ of $x$ there is an arcwise connected open neighbourhood $V$ of $x$ contained in $U$.

A metric tree is a uniquely arcwise connected geodesic metric space.

It is clear that any metric tree is a topological tree. The main result of [11] states that the converse holds too.

Theorem 4.2 ([11], Thm. 5.1). $X$ is a topological tree if and only if $X$ is homeomorphic to a metric tree.

In connection with the uniqueness of signatures result by Hambly and Lyons [8], the following notion is needed.

Let $\gamma:[a, b] \rightarrow X$ be a rectifiable path in a metric space $X . \gamma$ is called tree-like if there exists a metric tree $T$ and Lipschitz maps $\psi: T \rightarrow X$ and $\phi:[a, b] \rightarrow T$ such that $\phi(a)=\phi(b)$ and $\psi \circ \phi$ is a reparametrization of $\gamma$.

Equivalent characterizations of this property are given in Theorem 6.7.1 of [10], among these is the original definition of [8].

An equivalence relation on the space of rectifiable paths in $X$ is defined by

$$
\gamma \sim \mu \Leftrightarrow \gamma * \bar{\mu} \text { is tree-like . }
$$

Here $\bar{\mu}$ is a backwards parametrization of $\mu$. In this definition it is understood that the paths are translated so that the end point of $\gamma$ agrees with the start point of $\bar{\mu}$.

Let $S\left(\mathbb{R}^{n}\right) \subset \prod_{i=0}^{\infty}\left(\mathbb{R}^{n}\right)^{\otimes i}$ be the set of signatures of rectifiable paths in some fixed $n \in \mathbb{N}$. Given such a path $\gamma:[0,1] \rightarrow \mathbb{R}^{n}$, its signature is

$$
S_{\infty}(\gamma)_{0,1}:=\left(1, \int_{0<t<1} \mathrm{~d} \gamma_{t}, \ldots, \int_{0<t_{1}<\cdots<t_{k}<1} \mathrm{~d} \gamma_{t_{1}} \otimes \cdots \otimes \mathrm{d} \gamma_{t_{k}}, \ldots\right) \in \prod_{i=0}^{\infty}\left(\mathbb{R}^{n}\right)^{\otimes i},
$$

Also here Chen's identity (4.1) holds, and thus $S\left(\mathbb{R}^{n}\right)$ naturally has a group structure $\left(S\left(\mathbb{R}^{n}\right), \otimes, \mathbf{1}\right)$, where $\mathbf{1}$ is the element $(1,0,0, \ldots)$ in $\prod_{i=0}^{\infty}\left(\mathbb{R}^{n}\right)^{\otimes i}$. The main result of Hambly and Lyons $[8]$ is the following. 
Theorem 4.3 ([8], Thm. 4). Given two paths $\gamma$ and $\mu$ in $\mathbb{R}^{n}$ it holds that $S(\gamma)=S(\mu)$ if and only if $\gamma \sim \mu$.

In particular, every equivalence class contains up to translations and reparametrizations a unique path of shortest length. These are called tree-reduced. Injective paths for example are always tree-reduced. In [2] there is an extension of this uniqueness theorem to weakly geometric rough paths in Banach spaces.

As a consequence of the results stated so far, the inverse limit $G^{\infty}\left(\mathbb{R}^{n}\right)$ is a metric tree. Here are the details.

Theorem 4.4. There is a natural group isomorphism identifying the space of signatures $S\left(\mathbb{R}^{n}\right)$ with $G^{\infty}\left(\mathbb{R}^{n}\right)$, where $\left(G^{\infty}\left(\mathbb{R}^{n}\right), d_{\infty}, 1_{\infty}\right)$ is the inverse limit of the free nilpotent Lie groups $\left(G^{k}\left(\mathbb{R}^{n}\right), d_{k}, 1_{k}\right)$ with left invariant Carnot-Carathéodory metrics $d_{k}$ as defined in Section 2. Further, the metric space $G^{\infty}\left(\mathbb{R}^{n}\right)$ is a complete metric tree.

Proof. Set $\left(X_{k}, \star_{k}\right):=\left(G^{k}\left(\mathbb{R}^{n}\right), 1_{k}\right)$ for all $k \in \mathbb{N}$ with the metric and projections defined earlier for $G^{k}\left(\mathbb{R}^{n}\right)$. Now $\left(x_{1}, x_{2}, \ldots\right) \in X_{\infty}$ if and only if $\pi_{k+1}\left(x_{k+1}\right)=x_{k}$ for all $k \geq 1$ and $\sup _{k \in \mathbb{N}}\left\|x_{k}\right\|<\infty$. Let $\xi: X_{\infty} \rightarrow \prod_{i=0}^{\infty}\left(\mathbb{R}^{n}\right)^{\otimes i}$ be the map given by

$$
\xi\left(x_{1}, x_{2}, \ldots\right):=\left(1, \xi_{1}\left(x_{1}\right), \xi_{2}\left(x_{2}\right), \ldots\right) .
$$

We claim that $\xi$ is a group isomorphism onto $S\left(\mathbb{R}^{n}\right)$. First if $x \in \prod_{i=0}^{\infty}\left(\mathbb{R}^{n}\right)^{\otimes i}$ is the signature $S_{\infty}(\gamma)_{0,1}$ of a rectifiable path $\gamma:[0,1] \rightarrow \mathbb{R}^{n}$, then

$$
\left\|S_{k}(\gamma)_{0,1}\right\| \leq L\left(t \mapsto S_{k}(\gamma)_{0, t}\right)=L(\gamma)<\infty
$$

and hence $x$ is in the image of $\xi$. On the other side assume that $x=\xi\left(x_{\infty}\right)$ for some $x_{\infty} \in X_{\infty}$. Because each $\pi_{k}$ has the continuous path lifting property and each $X_{k}$ is proper and geodesic by Lemma 4.1, it follows from Proposition 2.9 that $X_{\infty}$ is a geodesic metric space. So let $\gamma:[0,1] \rightarrow X_{\infty}$ be a path of finite length with $\gamma(0)=1_{\infty}$ and $\gamma(1)=x_{\infty}$. Because each $\pi_{1}^{k}: X_{k} \rightarrow X_{1}=\mathbb{R}^{n}$ has the unique path lifting property with lifts given by the step $i$ signature of a path, it holds that $x_{k}=S_{k}\left(\pi_{1}^{\infty} \circ \gamma\right)_{0,1}$ for all $k \in \mathbb{N}$. Thus $x=S_{\infty}\left(\pi_{1}^{\infty} \circ \gamma\right)_{0,1}$. It follows that $\xi\left(X_{\infty}\right)=S\left(\mathbb{R}^{n}\right)$. By construction it is clear that $\xi$ is injective. This implies that $\xi: X_{\infty} \rightarrow S\left(\mathbb{R}^{n}\right)$ is a bijection. By the construction of $\xi$, the group structure given on $X_{\infty}$ as defined in Lemma 3.1 and the fact that the truncation operator $S\left(\mathbb{R}^{n}\right) \rightarrow G^{k}\left(\mathbb{R}^{n}\right)$ is a group homomorphism it is not hard to check that $\xi: X_{\infty} \rightarrow S\left(\mathbb{R}^{n}\right)$ is a group homomorphism and because also $\xi$ is injective, it is a group isomorphism.

We already know from Proposition 2.9 that $X_{\infty}$ is a complete geodesic metric space. Next we show that $X_{\infty}$ is a metric tree. Assume that $\gamma_{1}, \gamma_{2}:[0,1] \rightarrow X_{\infty}$ are two injective rectifiable paths with the same start and end points. We want to show that $\operatorname{im}\left(\gamma_{1}\right)=\operatorname{im}\left(\gamma_{2}\right)$. For this both can be assumed to be parametrized proportionally to arc length (i.e., $L\left(\left.\gamma_{k}\right|_{[0, t]}\right)=L\left(\gamma_{k}\right) t$ for $k=1,2$ ). By Lemma 3.1, the space $X_{\infty}$ is a group and $d_{\infty}$ is left invariant and therefore we can assume that $\gamma_{1}(0)=\gamma_{2}(0)=1_{\infty}$. As obtained above, $\xi\left(\gamma_{k}(t)\right)=$ $S_{\infty}\left(\pi_{1}^{\infty} \circ \gamma_{k}\right)_{0, t}$ for $k=1,2$ and $t \in[0,1]$. Because $\gamma_{k}(t)$ is injective, the paths $\pi_{1}^{\infty} \circ \gamma_{k}$ in $\mathbb{R}^{n}$ are tree-reduced. Because $S_{\infty}\left(\pi_{1}^{\infty} \circ \gamma_{1}\right)_{0,1}=S_{\infty}\left(\pi_{1}^{\infty} \circ \gamma_{2}\right)_{0,1}$, the uniqueness of signatures implies that $\pi_{1}^{\infty} \circ \gamma_{1}$ and $\pi_{1}^{\infty} \circ \gamma_{2}$ have a common reparametrization. In particular $c=L\left(\gamma_{1}\right)=L\left(\gamma_{2}\right)$. Because $\pi_{1}^{\infty}: X_{\infty} \rightarrow X_{1}$ has the unique path lifting property by Lemma 2.7 it follows from Lemma 2.6 that $L\left(\left.\pi_{1}^{\infty} \circ \gamma_{1}\right|_{[0, t]}\right)=L\left(\left.\pi_{1}^{\infty} \circ \gamma_{2}\right|_{[0, t]}\right)=c t$ for all $t \in[0,1]$. Thus $\pi_{1}^{\infty} \circ \gamma_{1}=\pi_{1}^{\infty} \circ \gamma_{2}$ and therefore also $\gamma_{1}=\gamma_{2}$ again by Lemma 2.7. By approximation, $\operatorname{im}\left(\gamma_{1}\right)=\operatorname{im}\left(\gamma_{2}\right)$ can be shown for injective paths, not necessarily of finite length. This shows that $X_{\infty}$ is a metric tree.

As a consequence of Proposition 2.9 it follows that any tree-reduced path in $\mathbb{R}^{n}$ is the projection of a geodesic in $\left(G^{\infty}\left(\mathbb{R}^{n}\right), d_{\infty}\right)$. Together with the second part of Proposition 2.9 , the next result shows that paths in $\mathbb{R}^{n}$ can actually be approximated by projections of geodesics in some $G^{k}\left(\mathbb{R}^{n}\right)$.

Corollary 4.5. For any (continuous) path $\gamma:[0,1] \rightarrow \mathbb{R}^{n} \cong G^{1}\left(\mathbb{R}^{n}\right)$ and any $\epsilon>0$ there is some $k \geq 1$ and a geodesic $\gamma_{k}:[0,1] \rightarrow G^{k}\left(\mathbb{R}^{n}\right)$ such that $L\left(\gamma_{k}\right) \leq L(\gamma), \pi_{1}^{k}\left(\gamma_{k}(\delta)\right)=\gamma(\delta)$ for $\delta=0,1$ and

$$
\left\|\gamma-\pi_{1}^{k} \circ \gamma_{k}\right\|_{\infty}<\epsilon .
$$


Proof. Note first that $\gamma$ can be uniformly approximated by tree-reduced paths with length bounded from above by $L(\gamma)$ while fixing the end points. So without loss of generality we may assume that $\gamma$ already is a treereduced rectifiable path. Let $\left(G^{\infty}\left(\mathbb{R}^{n}\right), 1_{\infty}\right)$ be, as before, the inverse limit of the sequence of pointed metric spaces $\left(G^{k}\left(\mathbb{R}^{n}\right), 1_{k}\right)_{k \in \mathbb{N}}$. Since $\pi_{1}^{\infty}: G^{\infty}\left(\mathbb{R}^{n}\right) \rightarrow \mathbb{R}^{n}$ is surjective by Lemma 2.2 , there is a point $x_{\infty} \in G^{\infty}\left(\mathbb{R}^{n}\right)$ that projects to $\gamma(0) \in \mathbb{R}^{n}$. Due to Lemma $2.7, \pi_{1}^{\infty}$ has the unique path lifting property and hence $\gamma$ lifts to a path $\gamma_{\infty}:[0,1] \rightarrow G^{\infty}\left(\mathbb{R}^{n}\right)$ starting at $x_{\infty}$. Since $\gamma$ is tree-reduced, the path $\gamma_{\infty}$ is injective and hence by Theorem 4.4 it is a geodesic (not necessarily parametrized proportionally to arc length). Since $\gamma_{\infty}$ is the unique geodesic (up to reparametrizations) connecting its end points it now follows from Proposition 2.9 that there is an increasing sequence $\sigma: \mathbb{N} \rightarrow \mathbb{N}$ and geodesics $\gamma_{\sigma(k)}$ in $G^{\sigma(k)}\left(\mathbb{R}^{n}\right)$ for each $k \in \mathbb{N}$ such that $\pi_{1}^{\sigma(k)} \circ \gamma_{\sigma(k)}$ : $[0,1] \rightarrow G^{\sigma(k)}\left(\mathbb{R}^{n}\right) \rightarrow \mathbb{R}^{n}$ converges uniformly to $\pi_{1}^{\infty} \circ \gamma_{\infty}=\gamma$.

Instead of paths in $\mathbb{R}^{n}$, the analogous statement holds for paths in $G^{k}\left(\mathbb{R}^{n}\right)$ which can be approximated by projections of geodesics in $G^{i}\left(\mathbb{R}^{n}\right)$ for some $i>k$. The proof is essentially the same.

Remark 4.6. Although the projection $\pi_{1}^{\infty}: X_{\infty} \rightarrow X_{1}$ has the unique path lifting property if each $\pi_{i}$ has it by Lemma 2.7, the map $\pi_{1}^{\infty}$ in general does not inherit the continuous path lifting property. This can be seen from the sequence of rectifiable paths $\gamma_{n}:[0,2] \rightarrow \mathbb{R}^{2}$ given by

$$
\gamma_{n}(t):= \begin{cases}(1-t, 0) & \text { if } t \in[0,1] \\ \left(t-1, \frac{1}{n}(t-1)\right) & \text { if } t \in[1,2]\end{cases}
$$

and their lifts $\bar{\gamma}_{n}$ to $G^{\infty}\left(\mathbb{R}^{2}\right)$. Each $\bar{\gamma}_{n}$ is injective and because $\left(G^{\infty}\left(\mathbb{R}^{2}\right), d_{\infty}\right)$ is a metric tree by Theorem 4.4 , it holds that

$$
d_{\infty}\left(\bar{\gamma}_{n}(0), \bar{\gamma}_{n}(2)\right)=L\left(\bar{\gamma}_{n}\right) \geq L\left(\gamma_{n}\right) \geq 2 .
$$

But the limit path $\gamma:[0,2] \rightarrow \mathbb{R}^{2}$ has trivial signature, i.e., $\bar{\gamma}(0)=\bar{\gamma}(2)$ for its lift into the limit space $\left(G^{\infty}\left(\mathbb{R}^{2}\right), d_{\infty}\right)$. Thus $\pi_{1}^{\infty}: G^{\infty}\left(\mathbb{R}^{2}\right) \rightarrow \mathbb{R}^{2}$ does not have the continuous path lifting property.

\section{TREes AS TOPOLOGICAL GROUPS}

The main goal of this section is to prove that nontrivial topological trees cannot have the structure of a topological group. This is implied by the following theorem. As a consequence we obtain that right translations are not continuous in the limit space $G^{\infty}\left(\mathbb{R}^{n}\right)$, or more generally, the space $G^{\infty}\left(\mathbb{R}^{n}\right)$ is not homeomorphic to a topological group.

Theorem 5.1. Assume that $X$ is a topological tree with a group structure $\left(\cdot,{ }^{-1}, e\right)$ such that all left translations $x \mapsto g \cdot x$ and right translations $x \mapsto x \cdot g$ are continuous. If $X$ contains more than one point, then $X$ is homemorphic to $\mathbb{R}$.

Proof. We first show that in case $X$ is not an interval, then there cannot be a group structure on $X$ with continuous left and right translations. This is proved by contradiction. Due to Theorem 4.2 we assume that $X$ is a metric tree.

Claim 1: If $X$ is not an interval, it contains a tripod. This means that there are different points $x, x_{1}, x_{2}, x_{3} \in$ $X$ such that $\left[x, x_{i}\right] \cap\left[x, x_{j}\right]=\{x\}$ for $i \neq j$.

Proof of claim 1: Assume that $X$ does not contain a tripod. Fix some $x_{0} \in X$. Assume first that there is some $\epsilon>0$ for which the set $A_{\epsilon}:=\left\{x \in X: d\left(x_{0}, x\right)=\epsilon\right\}$ contains more than two points. Then any three points in $A_{\epsilon}$ form a tripod, contradicting the assumption. Now assume that $A_{\epsilon}$ contains only one point for any $\epsilon>0$. Then $f: X \mapsto \mathbb{R}$ given by $f(x):=d\left(x_{0}, x\right)$ is an isometric embedding of $X$ onto some interval in $\mathbb{R}$. Finally assume that there are two different points $x_{-}, x_{+} \in A_{\epsilon}$ for some $\epsilon>0$. Let $X_{+}$be the set of points $x \in X$ for which $\left[x_{0}, x_{+}\right] \cap\left[x_{0}, x\right] \neq\left\{x_{0}\right\}$. Because $X$ is uniquely arcwise connected this means that $\left[x_{0}, x_{+}\right] \cap\left[x_{0}, x\right]=\left[x_{0}, z\right]$ for some $z \neq x_{0}$. If now $x, y \in X_{+}$, then $\left[x_{0}, x\right]$ and $\left[x_{0}, y\right]$ meet in some initial segment 
and thus $\left[x_{0}, x\right] \cap\left[x_{0}, y\right]=\left[x_{0}, z\right]$ for some $z \neq x_{0}$ as before. But since $X$ does not contain a tripod, $x=z$ if $d\left(x_{0}, x\right) \leq d\left(x_{0}, y\right)$ and $y=z$ if $d\left(x_{0}, y\right) \leq d\left(x_{0}, x\right)$. Similarly we define $X_{-}$with respect to $x_{-}$. Note that $X_{+} \cap X_{-}$are disjoint, otherwise $\left[x_{0}, x_{+}\right] \cup\left[x_{0}, x_{-}\right]$would form a tripod. Thus $f: X \rightarrow \mathbb{R}$ defined by

$$
f(x):= \begin{cases}0 & \text { if } x=x_{0} \\ +d\left(x_{0}, x\right) & \text { if } x \in X_{+} \\ -d\left(x_{0}, x\right) & \text { if } x \in X_{-}\end{cases}
$$

is an isometric embedding of $X$ onto an interval of $\mathbb{R}$. This proves the claim.

So there exists a tripod in $X$ as stated in the claim above. Left (or right) translating these arcs by $x^{-1}$ we may assume that $x=e$ is the center of a tripod. Pick some point $y \in\left[e, x_{1}\right] \backslash\left\{e, x_{1}\right\}$. Note that since left translation by $y$ is a homeomorphism, any set $y \cdot I$ is an arc if and only if $I$ is an arc. Thus, $y \cdot\left[e, x_{i}\right]=\left[y, y \cdot x_{i}\right]$.

Claim 2: At most one of the $\operatorname{arcs}\left[y, y \cdot x_{1}\right],\left[y, y \cdot x_{2}\right]$ and $\left[y, y \cdot x_{3}\right]$ intersects the segment $[e, y] \backslash\{y\}$ and at most one of these arcs intersects $\left[y, x_{1}\right] \backslash\{y\}$.

Proof of claim 2: This claim is quite clear since if two different $\left[y, y \cdot x_{i}\right]$ and $\left[y, y \cdot x_{j}\right]$ intersect say $[e, y] \backslash\{y\}$, then $\left[y, y \cdot x_{i}\right] \cap\left[y, y \cdot x_{j}\right]=[y, z]$ for some $z \neq y$. But this contradicts that $\left[y, y \cdot x_{i}\right] \cap\left[y, y \cdot x_{j}\right]=\{y\}$.

So there exists some $i \in\{1,2,3\}$ and a sequence $\left(y_{n}\right)_{n \in \mathbb{N}}$ in $\left[e, x_{1}\right] \backslash\left\{e, x_{1}\right\}$ with $\lim _{n \rightarrow \infty} y_{n}=e$ with $\left[y_{n}, y_{n}\right.$. $\left.x_{i}\right] \cap\left[e, x_{1}\right]=\left\{y_{n}\right\}$. It follows that $\left[x_{i}, e\right] \cup\left[e, y_{n}\right] \cup\left[y_{n}, y_{n} \cdot x_{i}\right]$ is an arc. Set $U:=X \backslash\{e\}$ Because $X$ is locally arcwise connected, there exists some arcwise connected open neighbourhood $V$ of $x_{i}$ contained in $U$. Because right translation by $x_{i}$ is continuous, $y_{n} \cdot x_{i} \in V$ for some large enough $n$. For such an $n$ there exists an arc entirely in $V$ connecting $x_{i}$ with $y_{n} \cdot x_{i}$. But this contradicts that $\left[x_{i}, e\right] \cup\left[e, y_{n}\right] \cup\left[y_{n}, y_{n} \cdot x_{i}\right]$ is another arc connecting $x_{i}$ with $y_{n} \cdot x_{i}$.

This shows that $X$ is homeomorphic to an interval. If $X$ has more than one point, then there is a point in $X$ with a neighbourhood homeomorphic to $\mathbb{R}$. Because left translations are homemorphisms of $X$ that act transitively we obtain that all points of $X$ have neighbourhoods homeomorphic to $\mathbb{R}$. As an interval $X$ has thus to be homeomorphic to $\mathbb{R}$.

The conclusion in the theorem above can be strengthened by saying that $(X, \cdot)$ and $(\mathbb{R},+)$ are isomorphic as topological groups. This boils down to the fact that the only topological group structure on $\mathbb{R}$ is the standard one.

\section{REFERENCES}

[1] L. Ambrosio and B. Kirchheim, Currents in metric spaces. Acta Math. 185 (2000) 1-80.

[2] H. Boedihardjo, X. Geng, T. Lyons and D. Yang, The signature of a rough path: Uniqueness. Adv. Math. 293 (2016) $720-737$.

[3] D. Burago, Y. Burago and S. Ivanov, A course in metric geometry. Vol. 33 of Graduate Studies in Mathematics. American Mathematical Society, Providence (2001).

[4] K.T. Chen, Iterated integrals and exponential homomorphisms. Proc. Lond. Math. Soc. 4 (1954) 502-512.

[5] K.T. Chen, Integration of paths - a faithful representation of paths by non-commutative formal power series. Trans. Am. Math. Soc. 89 (1958) 395-407.

[6] J. Cheeger and B. Kleiner, Realization of metric spaces as inverse limits, and bilipschitz embedding in $L_{1}$. Geom. Funct. Anal. 23 (2013) 96-133.

[7] P. Friz and N. Victoir, Multidimensional Stochastic Processes as Rough Paths. Vol. 120 of Cambridge Studies in Advanced Mathematics. Cambridge University Press, Cambridge (2010).

[8] B. Hambly and T. Lyons, Uniqueness for the signature of a path of bounded variation and the reduced path group. Ann. Math. 171 (2010) 109-167.

[9] E. Helly, Über lineare Funktionaloperationen. Sitzsber. Ahad. Wiss. Wien 121 (1912) 265-297.

[10] T. Lévy, The master field on the plane. Astérisque 388 (2017) ix +201.

[11] J.C. Mayer and L.G. Oversteegen, A topological characterization of R-trees. Trans. Am. Math. Soc. 320 (1990) $395-415$.

[12] L.C. Young, An inequality of the Hölder type, connected with Stieltjes integration. Acta Math. 67 (1936) $251-282$. 